\title{
biomolecules
}

ISSN 2218-273X

www.mdpi.com/journal/biomolecules/

Article

\section{Skin Immunization Obviates Alcohol-Related Immune Dysfunction}

\author{
Rhonda M. Brand ${ }^{1,2,3, *}$, John Mark Stottlemyer ${ }^{1}$, Rachel A. Cline ${ }^{1}$, Cara Donahue ${ }^{1}$, \\ Jaideep Behari ${ }^{2}$ and Louis D. Falo, Jr. $1,4,5,6,7$
}

1 Department of Dermatology, School of Medicine, University of Pittsburgh, Pittsburgh, PA 15213, USA; E-Mails: jms176176@gmail.com (J.M.S.); rachel.a.cline@gmail.com (R.A.C.); cmdst36@pitt.edu (C.D.); lof2@pitt.edu (L.D.F.)

2 Division of Gastroenterology, Hepatology and Nutrition, Department of Medicine, School of Medicine, University of Pittsburgh, Pittsburgh, PA 15213, USA;

E-Mail: jab31@pitt.edu

3 Magee Women's Research Institute, Pittsburgh, PA 15213, USA

4 Department of Bioengineering, University of Pittsburgh, Pittsburgh, PA 15213, USA

5 Clinical and Translational Science Institute, School of Medicine, University of Pittsburgh, Pittsburgh, PA 15213, USA

6 The University of Pittsburgh Cancer Institute, Pittsburgh, PA 15232, USA

7 The McGowan Institute for Regenerative Medicine, School of Medicine, University of Pittsburgh, Pittsburgh, PA 15219, USA

* Author to whom correspondence should be addressed; E-Mail: rmb91@pitt.edu; Tel.: +1-412-648-3399; Fax: +1-412-383-6393.

Academic Editors: Natalia Osna and Kusum Kharbanda

Received: 9 July 2015 / Accepted: 30 September 2015 / Published: 6 November 2015

\begin{abstract}
Alcoholics suffer from immune dysfunction that can impede vaccine efficacy. If ethanol (EtOH)-induced immune impairment is in part a result of direct exposure of immune cells to $\mathrm{EtOH}$, then reduced levels of exposure could result in less immune dysfunction. As alcohol ingestion results in lower alcohol levels in skin than blood, we hypothesized that the skin immune network may be relatively preserved, enabling skin-targeted immunizations to obviate the immune inhibitory effects of alcohol consumption on conventional vaccines. We employed the two most common chronic EtOH mouse feeding models, the liver-damaging Lieber-DeCarli (LD) and liver-sparing Meadows-Cook (MC) diets, to examine the roles of $\mathrm{EtOH}$ and/or EtOH-induced liver dysfunction on alcohol related immunosuppression. Pair-fed
\end{abstract}


mice were immunized against the model antigen ovalbumin (OVA) by DNA immunization or against flu by administering the protein-based influenza vaccine either systemically (IV, IM), directly to liver (hydrodynamic), or cutaneously (biolistic, ID). We measured resulting tissue EtOH levels, liver stress, regulatory T cell (Treg), and myeloid-derived suppressor cell (MDSC) populations. We compared immune responsiveness by measuring delayed-type hypersensitivity (DTH), antigen-specific cytotoxic T lymphocyte (CTL), and antibody induction as a function of delivery route and feeding model. We found that, as expected, and independent of the feeding model, EtOH ingestion inhibits DTH, CTL lysis, and antigen-specific total IgG induced by traditional systemic vaccines. On the other hand, skin-targeted vaccines were equally immunogenic in alcohol-exposed and non-exposed subjects, suggesting that cutaneous immunization may result in more efficacious vaccination in alcohol-ingesting subjects.

Keywords: skin immunity; vaccination; alcohol; ethanol; intradermal immunization; skin; Lieber-DeCarli; Meadows-Cook

\section{Introduction}

Chronic alcohol abuse increases viral [1-5] and bacterial [6-8] infection rates, impairs host immune responses, and can augment co-incident disease progression leading to greater morbidity and mortality. Further, the effectiveness of preventive interventions such as vaccines against hepatitis B $[9,10]$, pneumococcal disease [11], and tuberculosis [12] is reduced in the setting of excessive alcohol use, contributing to observed increases in the frequency and severity of infections.

In attempts to improve the efficacy of traditional intramuscular (IM) vaccinations in alcoholics, high dose and accelerated IM vaccination schedules have been attempted and show some success in improving seroconversion rates in alcohol-consuming non-responders $[13,14]$. As a potential alternative, intradermal (ID) vaccination has been shown to be dose-sparing and can enhance responses in people who have failed traditional IM vaccinations [15,16]. ID immunization targets the skin immune system, including skin dendritic cells (DCs) and can induce both humoral and cellular immunity, including viral-clearing cytotoxic T lymphocyte (CTL) responses [17]. Since the magnitude of alcohol-induced immune suppression is both dose-related [18-20] and tissue-specific [21], skin, an organ with lower alcohol exposure [22], is only moderately affected by alcohol consumption compared to other immune tissues $[23,24]$. Thus, skin-targeted immunization could result in more efficacious vaccines in alcoholic patients by taking advantage of low skin alcohol levels [22] and an extensive skin DC network.

Modeling the effects of human chronic alcohol consumption remains problematic. Multiple animal models of alcohol exposure are in use, with considerable design variability, including the use of different animal models (including mouse strain), the form and route of EtOH administered, and the amount and duration of feeding; all of which lead to inconsistent results [25]. To evaluate the relative immunogenicity of skin immunization in the context of alcohol ingestion, we chose two commonly employed models differing significantly in the amount of liver damage induced. We reasoned that this approach would address the issue of skin immunogenicity in the setting of alcohol consumption, and the potential impact of concomitant liver dysfunction. Specifically we studied the immunologic impact of chronic 
alcohol exposure through the relatively "liver sparing" Meadows-Cook (MC) and "liver impairing" Lieber-DeCarli (LD) diets.

It has been established that C57/BL6 mice consume similar quantities of EtOH with both feeding regimes [25]. The $\mathrm{MC}$ model incorporates $20 \% \mathrm{EtOH}$ into drinking water and is combined with standard chow. It is essentially an extended low-stress feeding regime that is associated with very mild to unobservable steatosis and does not increase liver weight or alter corticosteroid levels [26,27]. On the other hand, LD incorporates $4 \%-6 \%$ EtOH with a nutritionally balanced liquid diet that includes 35\% dietary fat. These animals are pair-fed with control mice receiving an isocaloric liquid diet with dextrin maltose replacing EtOH. The LD diet causes moderate liver damage including increased liver weight and steatosis [28]. In this model, EtOH consumption generates free radicals and reactive oxygen species (ROS) in the liver, as a direct result of alcohol metabolism and indirectly as a result of gut barrier function impairment [29]. Alcohol consumption inhibits the tight junction proteins occludin and zona occluden, which are critical to the maintenance of intestinal barrier function, which leads to greater permeation of bacterial endotoxin into the circulation [30,31]. Endotoxin can activate Kupffer cells through TLR4 receptor binding [32], which results in secretion of the inflammatory cytokine TNF- $\alpha$, and increased oxidative stress that is reflected in increased ROS and lipid peroxidation, and decreased hepatocellular antioxidant activity, all of which contribute to steatosis and immune system impairment [33,34].

By utilizing and comparing these two models we sought to determine whether regional immunization targeted to the skin could obviate alcohol induced immune inhibition resulting from direct effects of alcohol on immune function, and/or indirect immuno-modulation by alcohol-damaged liver. To this end, we directly compared immune responsiveness by assessing delayed type hypersensitivity (DTH), antigen-specific cytotoxic T lymphocyte (CTL) activity, and antigen-specific antibody production. Our results suggest that independent of alcohol feeding method, skin immunization obviates alcohol-induced immune suppression observed with systemic immunization, suggesting a novel strategy to improve vaccine effectiveness in alcoholic patients.

\section{Results}

\subsection{EtOH Levels Are Significantly Less in Skin than in Blood}

We previously demonstrated that EtOH administration to rats through either acute (gavage) [35] and/or chronic (LD) feeding regimes [22] leads to significantly greater EtOH levels in blood than in skin. To extend these results to mice, we measured and compared blood and skin EtOH concentrations in groups of mice fed either LD or MC diets. For both feeding regimes, skin EtOH levels are significantly lower than blood levels, supporting the hypothesis that low skin EtOH levels could correlate with relative sparing skin immune function (Table 1). C57/BL6 mice consume similar quantities of EtOH with both feeding regimes [25], thus lower serum levels observed in MC-fed mice may reflect more rapid clearance or earlier cessation of feeding compared to LD (Table 1).

Table 1. Alcohol levels vary regionally.

\begin{tabular}{ccccc}
\hline Diet & LD EtOH & MC EtOH & LD Control & MC Control \\
\hline Serum EtOH (\%) & $0.1380 \pm 0.0251^{\mathrm{a}, \mathrm{b}}$ & $0.0244 \pm 0.0099^{\mathrm{a}, \mathrm{c}}$ & $0.0007 \pm 0.0006^{\mathrm{b}}$ & $0.0005 \pm 0.0003^{\mathrm{c}}$ \\
Skin EtOH (\%) & $0.0070 \pm 0.0017^{\mathrm{d}}$ & $0.0036 \pm 0.0014^{\mathrm{e}}$ & $0.0006 \pm 0.0002^{\mathrm{d}}$ & $0.0005 \pm 0.0003^{\mathrm{e}}$ \\
\hline \multicolumn{5}{c}{${ }^{\mathrm{a}} p<0.05,{ }^{\mathrm{b}} p<0.0001,{ }^{\mathrm{c}} p<0.05,{ }^{\mathrm{d}} p<0.0001,{ }^{\mathrm{e}} p<0.05}$.
\end{tabular}




\subsection{Alcohol Feeding Protocols Differentially Induce Steatohepatitis and Oxidative Stress}

a)

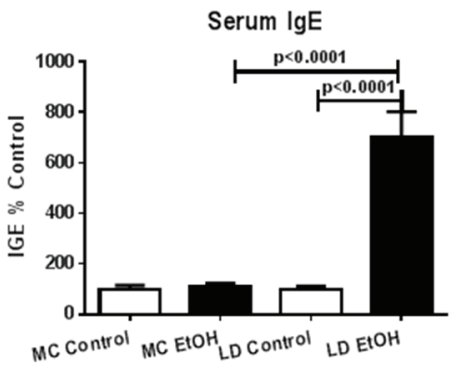

c)

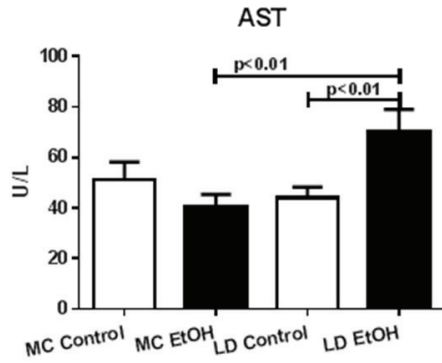

e)

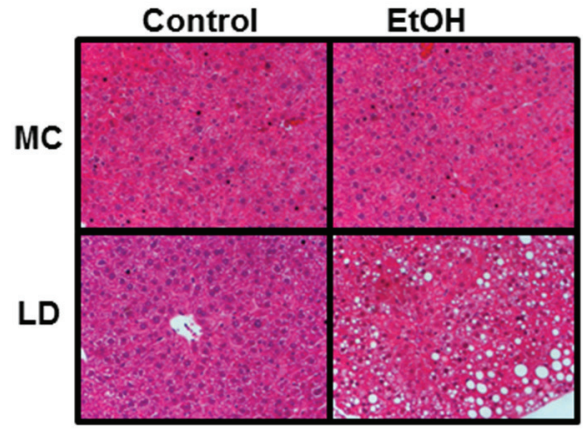

h)

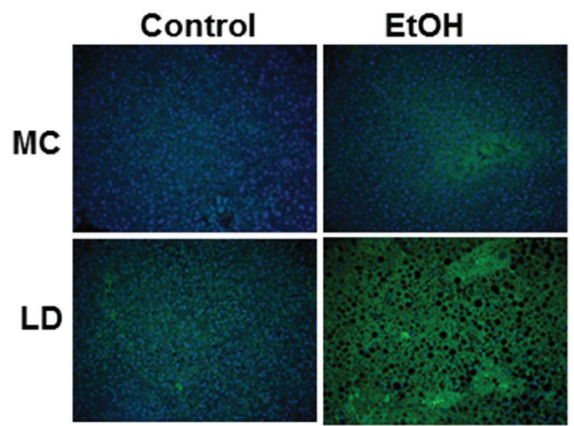

b)

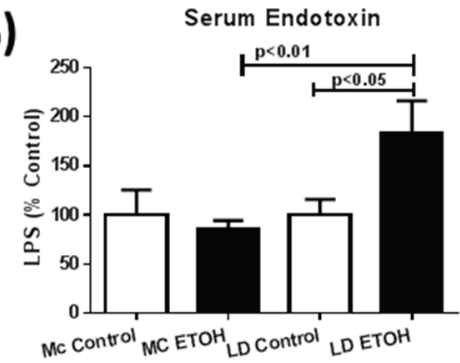

d)

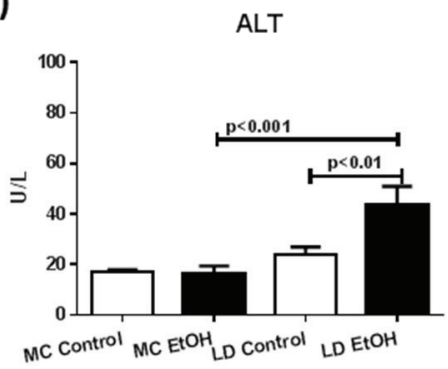

f)
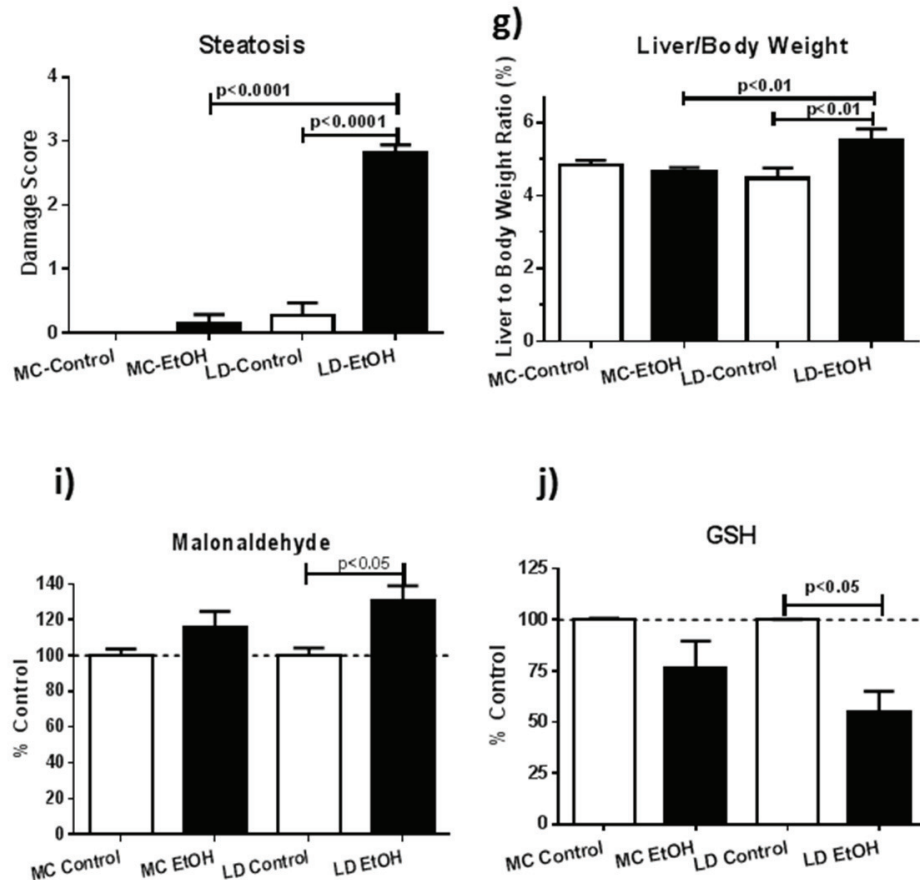

j)

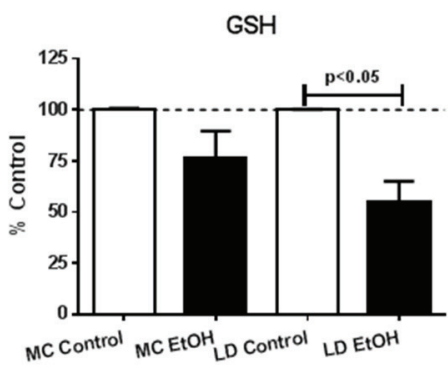

Figure 1. LD EtOH feeding causes greater steatohepatitis and oxidative damage than MC EtOH feeding. Mice were fed alcohol using MC or LD diets. (a) Non-antigen specific IgE is increased only after LD EtOH ingestion; (b) serum endotoxin levels are elevated only after LD EtOH exposure ( $n=7-10)$; (c) serum AST and (d) ALT are elevated after LD but not $\mathrm{MC} \mathrm{EtOH} \mathrm{exposure}(n=6)$. Livers were weighed and histological sections examined for visual changes due to the feeding models. Representative liver sections stained with $\mathrm{H} \& \mathrm{E}(\mathbf{e})$ demonstrate quantitatively more steatosis with LD than MC feeding (f) $(n=7-11)$; (g) liver weights as $\%$ of total body weight are increased after LD EtOH feeding $(n=7-13)$ and lipid peroxidation (4-hydroxynoneal staining) (h) is elevated as evidenced by immunofluorescence; (i) TBAR assay confirms elevated malonaldehyde in liver homogenates $(n=3-8)$; and (j) the antioxidant GSH is significantly depleted after LD $\operatorname{diet}(n=3-8)$. 
In initial studies we evaluated steatohepatitis and oxidative stress in animals fed LD vs. MC diets. We found that non-specific IgE levels indicative of liver damage [36] were elevated from LD but not MC feeding (Figure 1a). Further, serum LPS levels were elevated in LD not MC, indicative of intestinal barrier damage (Figure 1b). In mice fed LD but not MC diets, we found elevated liver enzymes (AST, ALT) (Figure 1c,d), increased liver to body weight ratio (\%) (Figure 1g), and histologically-confirmed steatohepatitis (Figure 1e,f) - all direct indicators of liver damage. EtOH metabolism in the liver, including generation of the alcohol metabolite acetaldehyde, generates reactive oxygen species (ROS) leading to oxidative stress [37]. Hydroxyl radicals cause lipid peroxidation, which correlates with levels of reactive malondialdehyde (MDA) and 4-hydroxynonenal (4HNE) [38]. In mice fed LD but not MC, immunohistochemistry specific for (4HNE) demonstrated increased liver lipid peroxidation (Figure 1h), and was supported by direct MDA assay confirming significantly increased lipid peroxidation in the liver homogenate (Figure 1i). The antioxidant imbalance resulting from EtOH metabolism is counteracted by multiple natural antioxidants, including glutathione (GSH), the major non protein thiol present in cells [34]. Consistent with the generation of high levels of ROS, livers from LD fed mice contained less GSH than pair-fed controls (Figure 1j). In all, these results are consistent with previously reported data and support increased liver damage and oxidative stress associated with LD, but not MC EtOH feeding protocols.

\subsection{Increases in Myeloid Derived Suppressor Cell (MDSC) Populations Correlate with Alcohol Induced Oxidative Stress}

To begin to determine whether increases in oxidative damage observed with LD feeding impacted resident immune cell populations, we quantitated the presence of MDSC and Treg populations in liver, spleen, and peripheral blood leukocytes (PBL). MDSCs suppress effector T cells and regulatory $\mathrm{T}$ cell (Treg) populations and have been shown to be up-regulated by multiple factors, including activation of ROS, TLR receptors, STATs, NF- $\kappa \beta$, and iNOS, all of which can be induced by alcohol consumption $[37,39,40]$. MDSC include at least two phenotypically and functionally distinguishable sub-populations, including $\mathrm{CD} 11 \mathrm{~b}^{+} \mathrm{Gr} 1^{\text {int }}$ and $\mathrm{CD} 11 \mathrm{~b}^{+} \mathrm{Gr} 1^{\text {hi }}$ MDSC populations. These subtypes have been functionally characterized in splenocytes, peripheral blood lymphocytes [41], and liver [42]. Flow cytometry of single cell suspensions demonstrates that both the strongly inhibitory $\mathrm{CD} 11 \mathrm{~b}^{+} \mathrm{Gr} 1^{\mathrm{int}+}$ and mildly inhibitory CD $11 \mathrm{~b}^{+} \mathrm{Gr} 1^{\mathrm{hi+}}$ MDSC populations are increased after LD EtOH feeding in liver and spleen (Figure 2a,b). This is in the context of unchanged $\left(\mathrm{CD} 11 \mathrm{~b}^{+} \mathrm{Gr} 1^{\mathrm{hi}+}\right)$ or modestly decreased $\left(\mathrm{CD} 11 \mathrm{~b}^{+} \mathrm{Gr} 1^{\mathrm{int}+}\right)$ populations in PBL (Figure 2a,b). Not surprisingly, given the lack of ROS generated by MC, we did not detect significant changes in either MDSC population after $\mathrm{MC}$ EtOH feeding. Nor did we detect differences in $\mathrm{CD} 4^{+} \mathrm{CD} 25^{+} \mathrm{Foxp} 3^{+}$Treg populations after either EtOH feeding protocol (Figure 2c). The elevated MDSCs measured after LD, but not MC, correlate with increased reactive oxygen species and liver damage observed in our biochemical and histological analysis. 
a) MDSC-Cd11 $b^{+}$Grint LD

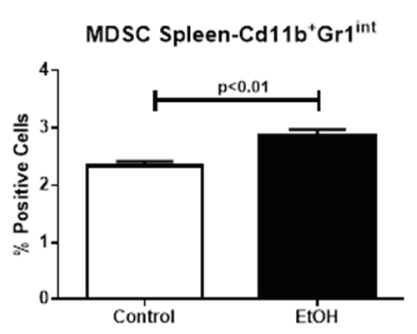

MDSC PBL-Cd11b ${ }^{+} \mathrm{Gr} 1^{\text {int }}$

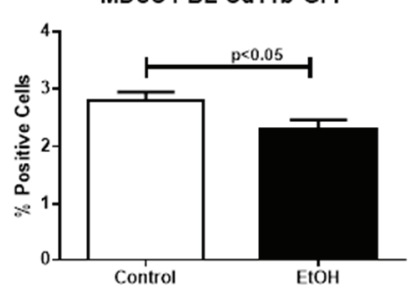

MDSC Liver-Cd11 b ${ }^{*}$ Gr $1^{\text {int }}$

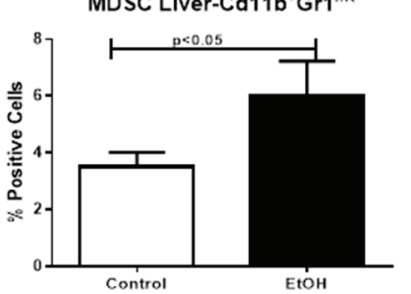

MC

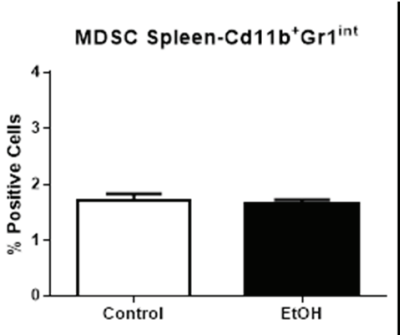

MDSC PBL-Cd11 $\mathrm{b}^{+} \mathrm{G} \mathbf{1}^{\mathrm{int}}$

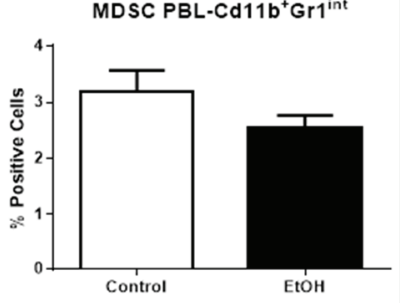

MDSC Liver-Cd11 ${ }^{+} G r 1^{\text {int }}$

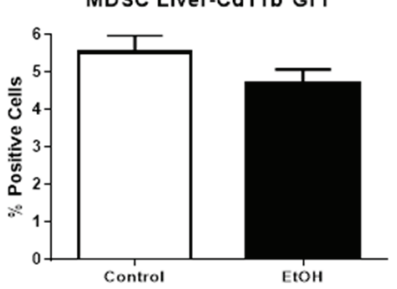

b)

LD MDSC-Cd11 $b^{+} G r^{h i}$

LD

MC
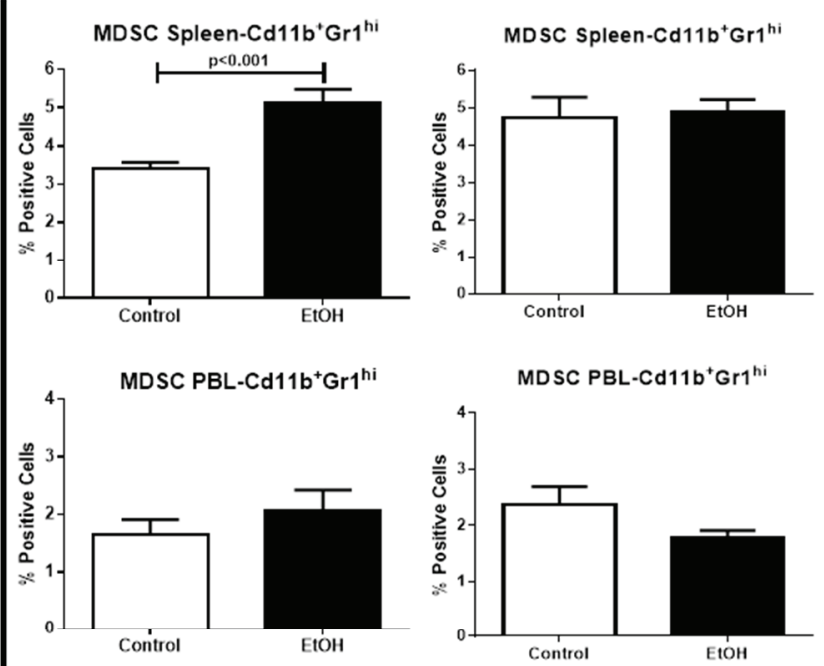

MDSC PBL-Cd11b ${ }^{+}$Gr1 ${ }^{\text {hi }}$
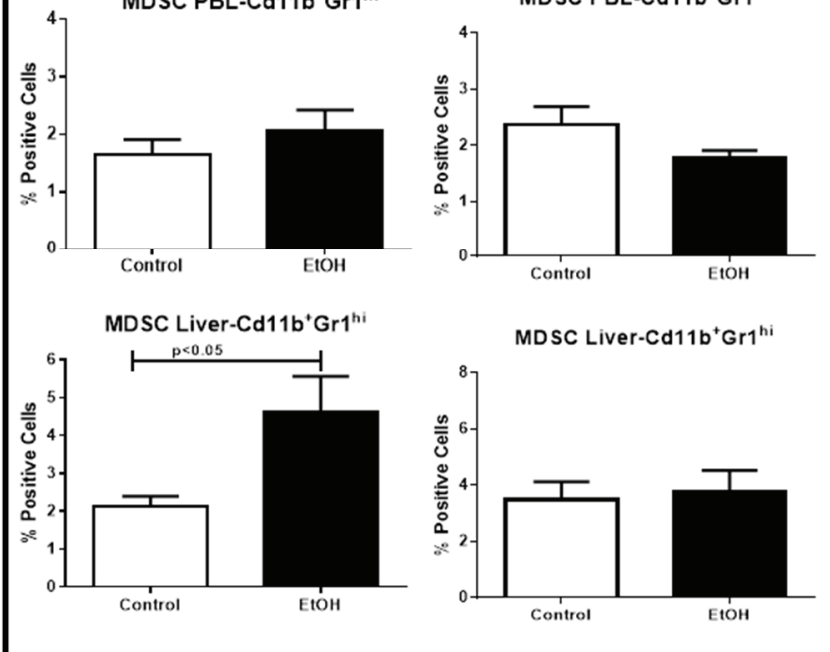

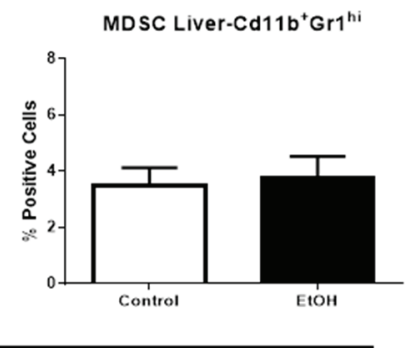

d) Representative Dot Plots

Treg-Cd4 ${ }^{+} \mathrm{C25}^{+}$Fox $\mathrm{P3}^{+}$ Gated on $\mathrm{Cd}^{2} 5^{+}$
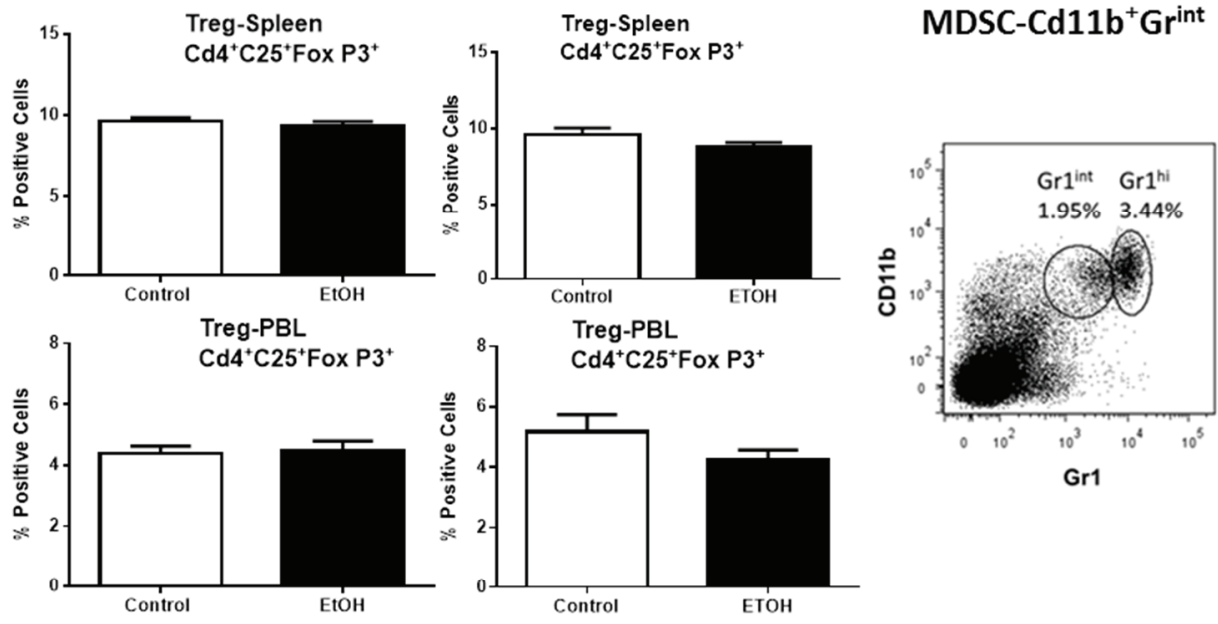

Gr1
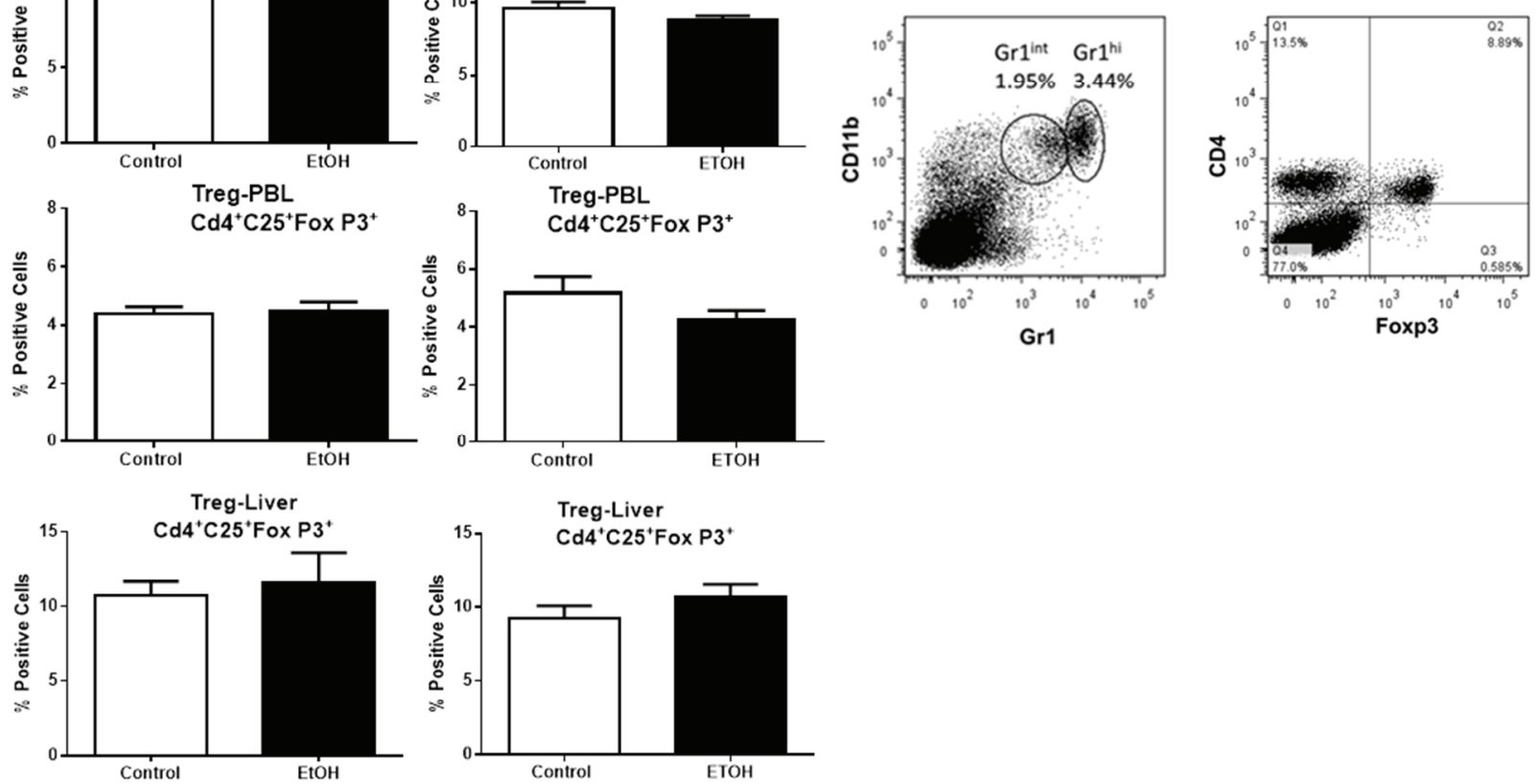

Figure 2. LD Alcohol Feeding Increases MDSC Populations in the Liver and Spleen. (a) LD EtOH feeding but not MC feeding induces $\mathrm{Cd} 11 \mathrm{~b}^{+} \mathrm{Gr} 1^{\text {int }}$ and (b) $\mathrm{Cd}_{1} 1 \mathrm{~b}^{+} \mathrm{Gr} 1^{\mathrm{hi}} \mathrm{MDSC}$ populations in the spleen and liver; (c) $\mathrm{Cd} 4^{+} \mathrm{Cd} 25^{+} \mathrm{Foxp} 3^{+}$Treg populations are unchanged by either EtOH feeding protocol; and (d) representative dot plots. 


\subsection{Skin Immunization Obviates Alcohol Associated DTH Inhibition}

\section{a) Systemic Immunization- IV and IM}
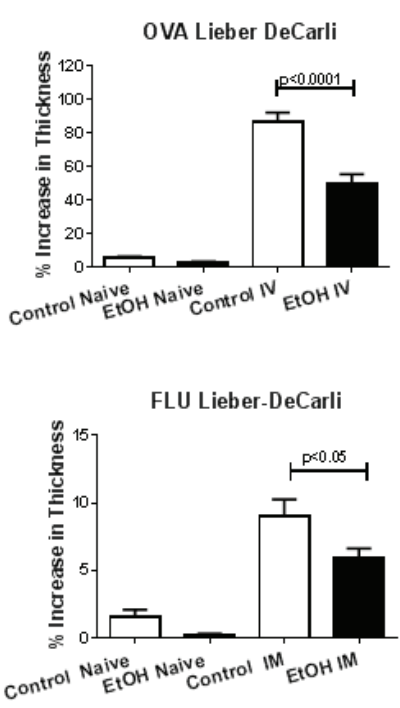

b) Liver Immunization- Hydrodynamic
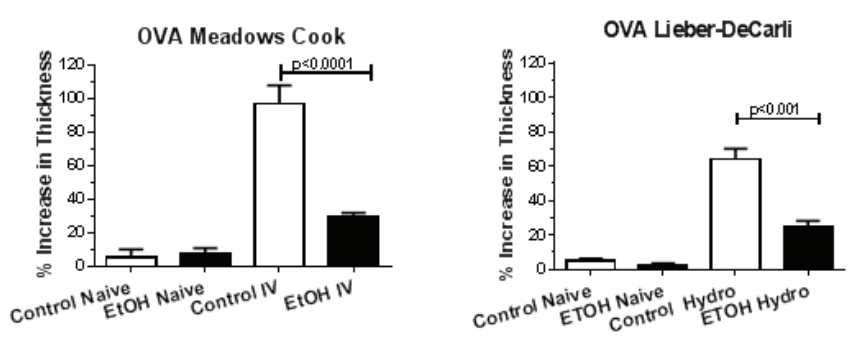

\section{c) Local Skin Immunization}
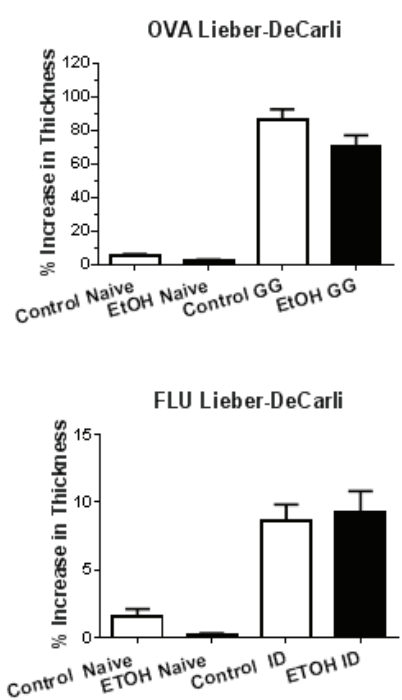

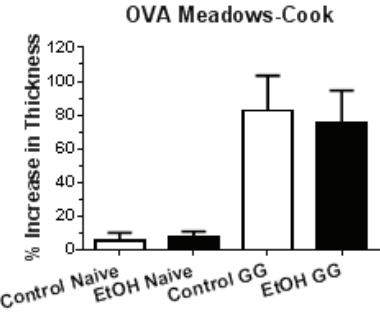

FLU Meadows-Cook

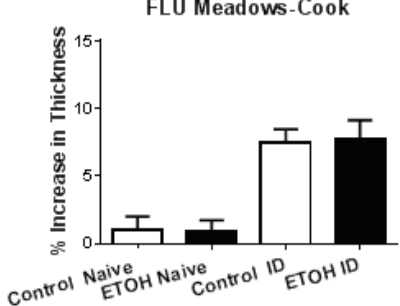

d) Representative H\&E Sections

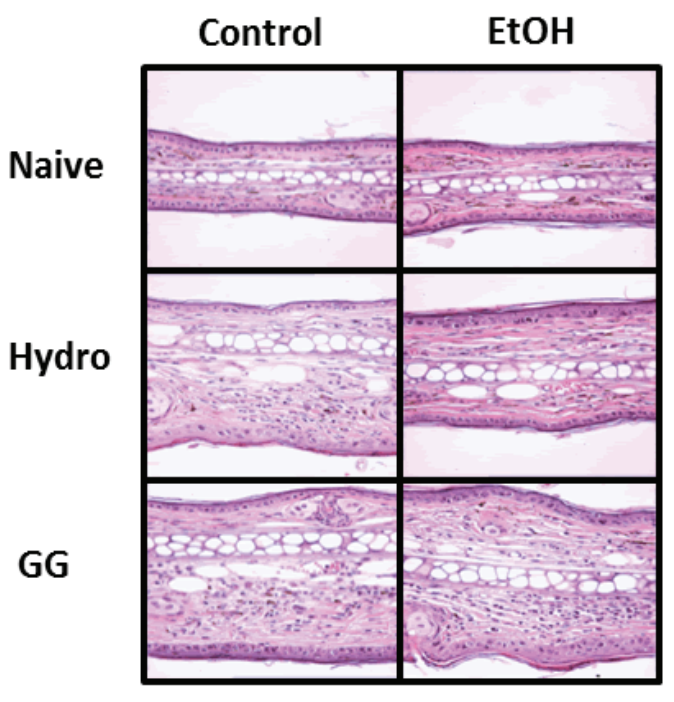

Figure 3. Skin Immunizations Obviate EtOH Induced DTH Inhibition. DTH responses are reported for groups of mice fed by either LD or MC ETOH feeding protocols as indicated after (a) systemic; (b) liver or (c) skin immunization with either OVA pDNA or FLU protein vaccine via intravenous, intramuscular, hydrodynamic, biolistic, or intradermal routes of administration as indicated. DTH responses were elicited and measured as ear or footpad swelling as defined in the methods section. Data are presented as \% increase in thickness (ear for OVA, footpad for FLU). LD and MC have equivalent DTH responses; (d) representative $\mathrm{H}$ and $\mathrm{E}$ stained ear sections after OVA immunization and elicitation.

We evaluated the delayed-type hypersensitivity (DTH) reaction as a measure of immune responsiveness to determine the impact of the route of antigen delivery on immune induction in alcohol-consuming mice. To accomplish this, groups of mice were immunized either with plasmid DNA encoding the model antigen 
Ovalbumin (OVA), or with the protein-based, clinically-relevant, and commercially-available Flu vaccine. Immunization routes included systemic immunization by the intramuscular (IM) or intravenous (IV) routes, or cutaneously by biolistic immunization (gene gun) or intradermal injection (ID). Further, to evaluate hepatic immune function, some groups of mice were immunized intrahepatically by hydrodynamic (HYDRO) injection, a DNA immunization method that results in antigen gene expression in hepatocytes [43]. Using these techniques, we found that DTH responses were inhibited following systemic (IV and IM) and hepatic immunization in animals consuming EtOH compared to non-EtOH consuming animals, regardless of the EtOH feeding protocol (Figure 3a,d). Further, hepatic immunization was substantially less effective in EtOH fed animals (Figure 3b,d). On the other hand, there was no significant difference in the DTH responses induced by skin immunization in EtOH consuming animals vs. non-EtOH consuming controls (Figure 3c,d). This was consistently true regardless of the EtOH feeding protocol, DNA vs. protein immunization, or GG vs. ID delivery. Thus, regardless of feeding protocols, reduced immunogenicity of traditional systemic protein and DNA vaccines observed in EtOH-consuming animals was not evident for skin-targeted immunizations.

\subsection{Skin Immunization Overcomes Alcohol Induced Inhibition of CTL Induction}

a) Skin vs IV-Systemic

LD

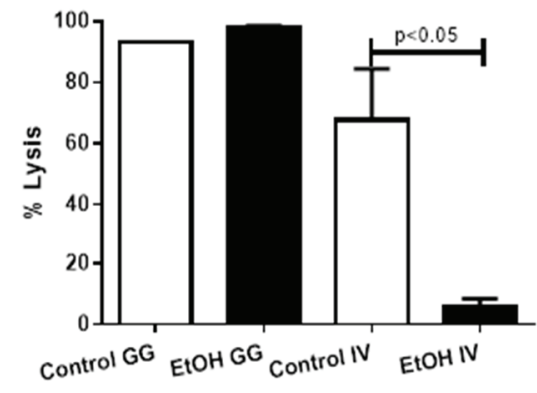

b) Skin vs IV-Systemic

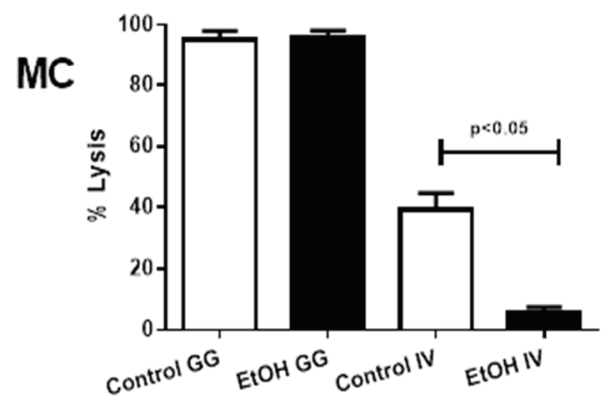

c) Skin vs IM-Systemic

e) Skin vs Hydro-Liver
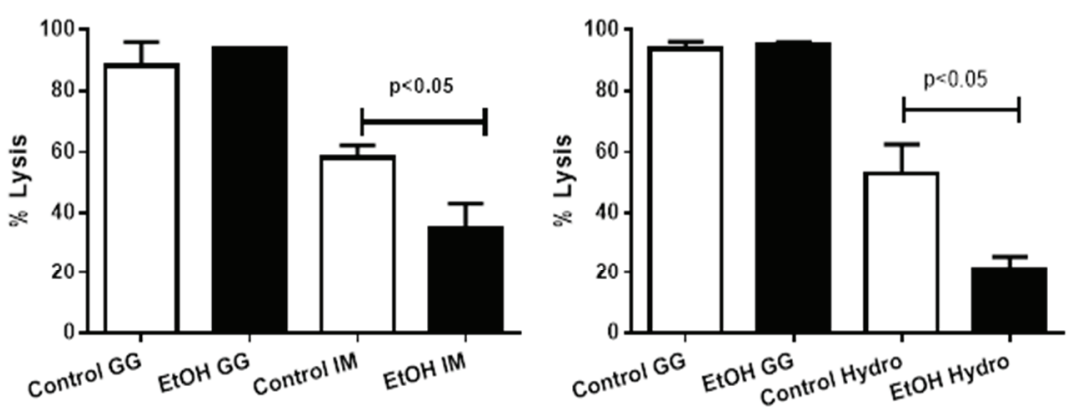

d) Skin vs IM-Systemic

f) Skin vs Hydro-Liver

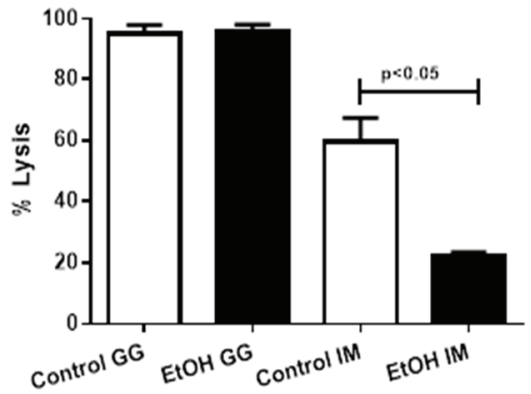

Figure 4. Skin Immunization Obviates EtOH Induced Inhibition of CTL Induction. Groups of animals fed ETOH by LD $(\mathbf{a}, \mathbf{c}, \mathbf{e})$ or $\mathrm{MC}(\mathbf{b}, \mathbf{d}, \mathbf{f})$ and their matched non-EtOH-fed controls were immunized by IV $(\mathbf{a}, \mathbf{b})$; IM $(\mathbf{c}, \mathbf{d})$; or HYDRO $(\mathbf{e}, \mathbf{f})$ routes as previously described and in each case compared to biolistically (GG) immunized paired EtOH-exposed and control mice biolistic. Immunization protocols were as previously described and CTL induction was determined by in vivo lytic activity and presented as \% lysis as defined in the methods section. Representative experiments are shown $(n=2-9)$. 
As alcoholics are more susceptible to infection and cancer, we sought to determine the effect of alcohol consumption on the induction of CTL responses by cutaneous or systemic vaccines, as measured by in vivo antigen-specific lytic activity. The induction of lytic responses was inhibited by EtOH consumption for vaccines delivered by IV, IM or hydrodynamic routes regardless of the method of EtOH feeding (Figure 4). In contrast, EtOH-consuming animals immunized cutaneously demonstrated strong antigen specific in vivo lytic activity that was equivalent to that observed in control EtOH naïve animals (Figure 4). This was consistent across feeding protocols. To ensure that potential EtOH effects were not masked by the high magnitude of antigen-specific lysis induced, we repeated skin immunizations without boosting, which resulted in more moderate degrees of lysis without differences between EtOH-consuming and naïve animals [44]. Thus, regardless of EtOH feeding protocols, EtOH associated inhibition of antigen-specific lytic cell induction was evident following systemic immunization, but avoided by skin-targeted antigen delivery.

\subsection{Skin Immunization Obviates Alcohol Induced Inhibition of Antigen-Specific IgG Induction}

LD

a) IM- Systemic

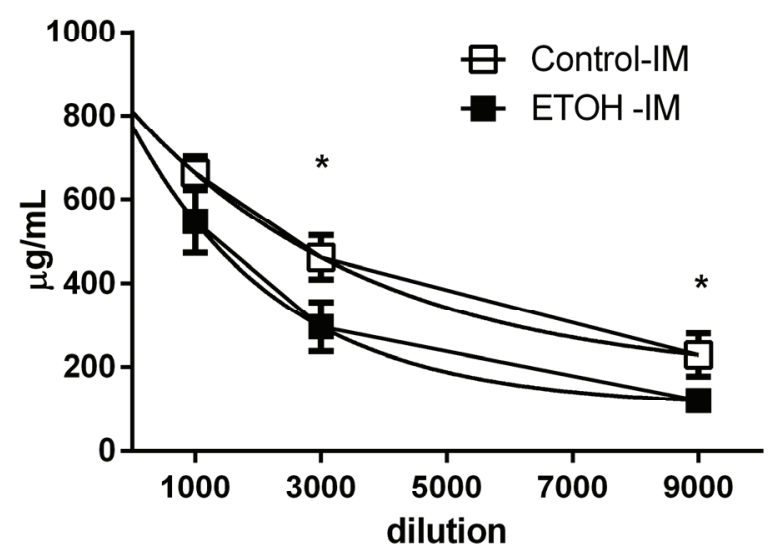

c) ID- Local Skin

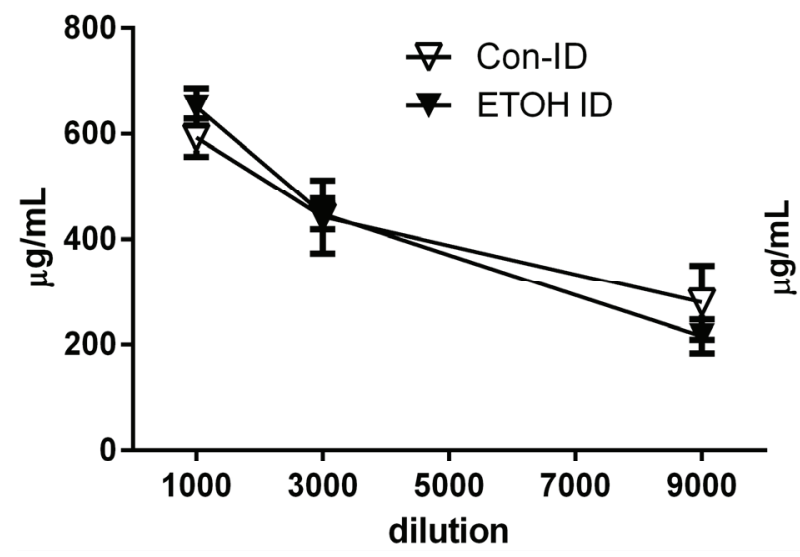

MC

b) IM- Systemic

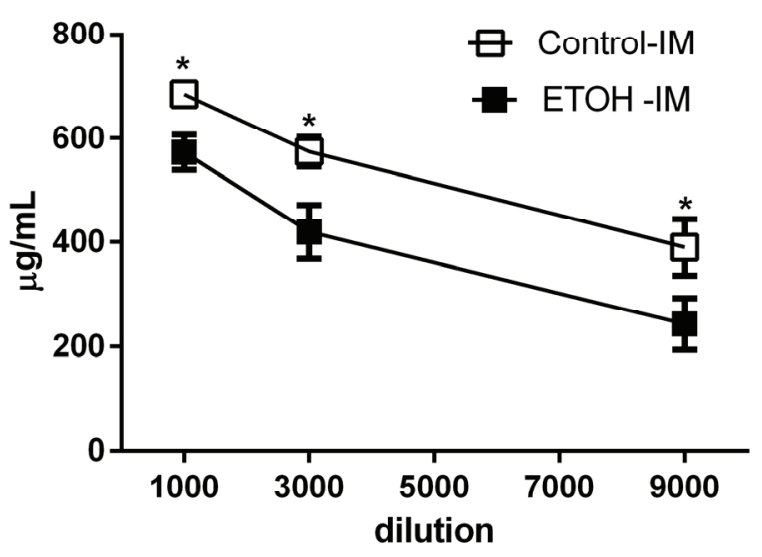

d) ID- Local Skin

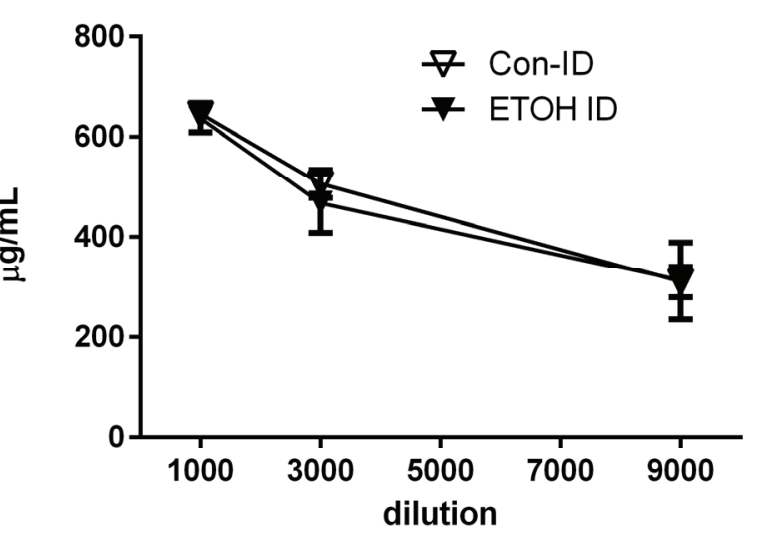

Figure 5. Skin Immunization Obviates EtOH Induced Inhibition of Vaccine Induced IgG. Mice fed ETOH by LD (a,c) or MC (b,d) protocols and EtOH-naïve controls were immunized with influenza vaccine by IM $(\mathbf{a}, \mathbf{b})$ or ID $(\mathbf{c}, \mathbf{d})$ routes and boosted on day $21(n=3-8)$. Total antigen-specific IgG, as determined by Elisa, is shown, with * $p<0.05$. 
EtOH ingestion is known to impair vaccine efficacy in humans, as measured by antibody responses including antigen-specific IgG [12]. Using influenza vaccine, we evaluated antigen specific IgG responses in both EtOH-consuming and EtOH-naïve groups of animals as a function of immunization route. EtOH consumption, whether by LD or MC protocols, significantly inhibited antigen-specific IgG responses elicited by IM immunization (Figure 5a,b). In contrast, there was not a significant difference between EtOH-consuming mice and naïve controls when the flu vaccine was delivered ID (Figure 5c,d). This was observed regardless of EtOH feeding protocol, suggesting that skin-targeted immunization can elicit effective $\mathrm{IgG}$ responses even in the setting of chronic alcohol ingestion.

\section{Discussion}

Considerable evidence from animal models and human studies suggests that chronic alcoholism is associated with clinically-relevant immune dysfunction [1-8]. Mechanisms of alcohol-induced immune suppression are complex, and depend on both direct effects of alcohol exposure on immune cells, as well as downstream effects of alcohol metabolism including, for example, the generation of acetaldehyde and ROS. Adding to this complexity are the myriad immunosuppressive effects of steatohepatitis and liver failure that are frequent sequelae of chronic alcoholism. Alcohol-related immune suppression is both tissue specific [21] and dose dependent [19,20]. We hypothesized that a significant component of immune inhibition resulting from alcohol consumption could be related to direct exposure of immune cells to alcohol and its metabolites. This is consistent with in vitro models demonstrating alcohol's inhibitory effects on dendritic cell function [45-47]. Given previous studies demonstrating relatively low levels of alcohol in skin compared to other organs [22,35], we reasoned that the skin immune system may be relatively spared from the immune-inhibitory effects of alcohol.

To test this directly, we compared the immunogenicity of traditional systemic vaccination strategies to skin targeted immunizations. Antigen was targeted to the systemic circulation (IV), muscle (IM), the liver (hydrodynamic delivery) or the skin (gene gun, ID), and immunogenicity was determined by evaluation of DTH, CTL, and humoral immune responses. Further, to more specifically address immune-suppressive effects from direct alcohol exposure $v s$. alcohol induced hepatic dysfunction, we studied these immunization strategies in models of chronic alcohol ingestion with, and without, hepatic dysfunction.

The potential effectiveness of skin vaccination in heavy alcohol users is supported by our results demonstrating that both DTH and CTL responses are inhibited by alcohol ingestion after systemic and hepatic immunization, but not after skin immunization. The skin immune system induces both the cellular and humoral arms of immunity, thereby allowing for the effective development of viral clearing CTL [17]. Our observation that alcohol ingestion inhibits DTH, CTL, and antibody responses independent of feeding model, is consistent with previous studies in both LD [48] and MC mice [49]. The observation that skin immunization overcomes alcohol inhibition in both models is encouraging for clinical extrapolation. To that end, inhibition of DTH responses by alcohol is well established in human subjects [50]. Kinetic studies using CTL and DTH assays reveal that immune suppression is strongest when alcohol ingestion occurs before or during sensitization [51-53], and may be reversible after EtOH cessation [54]. Further, in humans hemagglutinin inhibition of antibody following IM influenza vaccination is inhibited in patients with advanced liver cirrhosis [55], and alcohol dependency significantly reduces hepatitis B vaccine efficacy after IM delivery [56]. Finally, our results are consistent with reports that ID 
influenza vaccination is more effective than IM immunization in older adults [57] and immunosuppressed patients [58-60]. Thus, skin immunization has potential to effectively obviate poor seroconversion rates observed following IM vaccination in heavy alcohol users.

Mechanistically, it is well-established that alcohol interferes with the ability of DCs to activate T cells after antigen stimulation [61]. Alcohol interferes with DC maturation, resulting in decreased expression of the costimulatory surface molecules (CD40, CD80, CD86) important for T-cell activation [62,63]. It has also been reported that MHC class I and class II expression is decreased $[48,62]$, and that these differences are associated with reduced secretion of inflammatory cytokines such as TNF $\alpha$, IL-12, IFN $\gamma$, IL6 and IL-17A, and enhanced expression of IL-1 $\beta$, IL-10 and IL-13 by ethanol-exposed DCs $[48,53,64]$.

Alcohol effects on DC function also appear to be regional. Chronic alcohol ingestion decreases DC numbers in the spleen, but increases DCs in thymus [23]. Alcohol also induces hepatic DC migration to draining lymphoid tissue, but does not affect splenic DC migration. Hepatic DCs prime allogeneic cells more vigorously than splenic DCs after alcohol exposure. DC populations in the skin, with relatively low levels of alcohol exposure [22], appear to be only moderately affected by alcohol consumption. For example, four weeks of $20 \% \mathrm{EtOH}$ in mouse drinking water leads to a $30 \%$ reduction in Langerhans Cells (LCs), but no change in dermal dendritic cell (DDC) numbers. In contrast, total splenic DCs are reduced by $50 \%$ under the same conditions. Migration of LC and dermal DC from the skin to draining lymph nodes during inflammation is modestly delayed in alcohol-fed compared to control mice. LC are more sensitive to the effects of alcohol compared to dermal DC, as shorter durations of alcohol exposure are required to inhibit migration $[23,24,47]$. The relative sparing of dermal DC function may contribute to the preservation of skin immune function.

Tregs and MDSCs have inhibitory effects on antigen specific CTL induction and vaccine efficacy $[65,66]$. Previous studies suggest that MDSCs are increased in PBL after alcohol ingestion compared to normal controls [67]. Our data suggest that under non-pathological conditions, MDSC populations were increased in both the spleen and liver from the LD diet. The MC diet did not substantially alter MDSCs. Treg populations were not affected by either alcohol diet. The differences between MC and LD diets suggest that MDSC induction is likely an effect of alcohol associated liver dysfunction rather than a direct effect of alcohol itself. In any case, the minor changes observed did not appear to effect either alcohol associated immune suppression or the capacity of skin immunization to overcome it.

To address the impact of moderate liver damage on vaccination in mouse models of alcohol consumption, we directly compared the steatohepatitis-generating LD with the less-damaging MC diet. Direct comparisons between the two models are rarely performed. Histological and quantitative data demonstrate steatohepatitis after LD, but only minimal changes in liver morphology after MC. Our data suggests that only LD disrupts small intestine barrier function, as indicated by elevated serum LPS consistent with previous observations in mouse models [68] and humans [31]. All markers tested confirm extensive oxidative stress in LD, but not MC-fed mice, consistent with previously published studies [34,69,70]. The differences in inflammation and oxidative stress enabled us to address the impact of these stressors on immune function. These studies also provide a direct comparison of the liver damaging LD vs. liver-sparing MC diet on systemic immune responses in C57/BL6 mice, and suggest that alcohol induced immune suppression did not depend on increased serum LPS, steatohepatitis, or oxidative stress as measured by increased lipid peroxidation and decreased GSH in liver by LD, but not MC. Thus, in these models, alcohol-related immune suppression appears to be directly related to the direct effects of alcohol and its metabolites and not to indirect 
effects of hepatic dysfunction. It is important to note, however, that these mouse models correspond to moderate alcohol-induced liver damage, but do not model liver cirrhosis which may produce more severe immune dysfunction.

\section{Experimental Section}

\subsection{Feeding Regimes}

Female C57BL/6 mice (6-8 weeks old) were fed a nutritionally-adequate LD liquid diet [28] consisting of $18 \%$ protein, $37 \%$ fat, and $47 \%$ carbohydrate containing $5 \%(v / v) \mathrm{EtOH}(27 \%$ calories $)$, or a control diet in which EtOH is substituted isocalorically with dextrin maltose (Bio-Serve, Frenchtown, NJ, USA). EtOH was introduced gradually by increasing the content from $1 \%$ for two days to $3 \%$ for two days and then $5 \%(v / v)$ ethanol for the remainder of the experiment. Mice were given $5 \% \mathrm{EtOH}$ for one, four or eight weeks prior to immunization and remained on 5\% EtOH for the entire experiment.

A separate set of female C57/BL6 mice (6-8 weeks old) were provided EtOH in drinking water combined with chow ad libitum (MC). Mice were acclimatized to alcohol by ramping up the concentration starting at $10 \%$ for two days, increasing to $15 \%$ for five days and $20 \%$ for $6-8$ weeks at which time they were immunized and remained on $20 \% \mathrm{EtOH}$ for the duration of the experiment.

\subsection{Immunization Methods}

Ovalbumin pDNA was administered either via intramuscular (IM) or intravenous injection (IV), hydrodynamic injection (targeted to liver) or biolistic delivery (targeted to skin). The high pressure/high volume hydrodynamic immunization (Hydro, enables pDNA delivery directly into hepatocytes by rapid intravenous administration (6-8 s) of a large volume of DNA $(12 \mu \mathrm{g}$ of plasmid OVA DNA in $1.5 \mathrm{~mL}$ of PBS) [43]. Skin delivery was achieved with a biolistic gene gun system. Twelve $\mu$ g of plasmid OVA DNA was coated onto microscopic gold particles (1-3 $\mu \mathrm{m}$ in size), which were then delivered to the abdominal skin using a helium-powered GG (Helios, BioRad Laboratories, Hercules, CA, USA) at a helium pressure of 250 PSI (GG) [71]. For systemic delivery, we used IV or IM immunizations of $12 \mu \mathrm{g}$ of plasmid OVA DNA in $0.2 \mathrm{~mL}$ of PBS.

Fluvirin (207-2008 formulation, Novartis, East Hanover, NJ, USA) (FLU), a purified split virus preparation influenza vaccine, containing A/Wisconsin/67/205, A/Solomon Islands/3/200, and $\mathrm{B} /$ Malausia/2506/2004 hemagglutinin antigens (HA), was delivered to mice using IM or ID injections at a dose of $3 \mu \mathrm{g}$.

\subsection{Serum and Tissue Characterization}

Upon completion of immunization protocols, shaved skin (20 mg) was homogenized for 75 seconds in $300 \mu \mathrm{L}$ of PBS and spun for 10 minutes. We assayed the supernatant for skin EtOH with a L3K kit (Sekisui Diagnostics, Framingham, MA, USA). Serum EtOH, AST, ALT, and endotoxin were also determined with commercial kits (L3k, AST-SL, ALT-SL, Sekisui Diagnostics, 50-647U Lonza, Allendale, NJ, USA). Non-antigen-specific IgE levels were measured via ELISA (BD Opt EIA, BD Biosciences, San Jose, CA, USA). Data are presented as \% control for each feeding model and statistical 
significance determined with an ANOVA followed by Fisher Exact Test with significance set at $p<0.05$ (GraphPad Prism, GraphPad Software, Inc., La Jolla, CA, USA).

$\mathrm{H} \&$ E-stained paraffin embedded liver sections from non-immunized mice were evaluated for steatosis, by a blinded hepatologist, with $0=<5 \%, 1=5 \%-33 \%, 2=33 \%-66 \%, 3=>66 \%$ [72]. Statistical significance was determined with an ANOVA followed by Fisher Exact Test with significance set at $p<0.05$ (GraphPad Prism).

\subsection{Liver Biochemical Assays}

Liver samples were homogenized in $25 \mathrm{mM}$ HEPES, $1 \mathrm{mM}$ EGTA, $5 \mathrm{mM} \mathrm{MgCl} 2$ on ice and spun at 13k rpm for $15 \mathrm{~min}$ at $4{ }^{\circ} \mathrm{C}$ [73]. We tested supernatant for GSH (Gsh-Glo, Promega, Madison, WI, USA) and lipid peroxidation (Oxiselect TBARS Assay Kit, Cell BioLabs, San Diego, CA, USA). Protein levels were determined with Coomassie Plus Protein Reagent (Fisher Scientific, Pittsburgh, PA, USA). Data are normalized to $\mathrm{mg}$ protein and are presented as \% control diet. Statistical significance was determined with an ANOVA followed by Fisher Exact Test with significance set at $p<0.05$ (GraphPad Prism).

\subsection{HNE Immunohistochemistry}

Slides were deparafinized, rehydrated, washed in PBS, and stained with Polyclonal Goat anti 4-HNE primary antibody $1 / 250$ in 1\% BSA in PBS 0.1\% TritonX-100 (Alpha Diagnostic, San Antonio, TX, USA) overnight at $4{ }^{\circ} \mathrm{C}$, followed by conjugated Donkey anti-Goat IgG cy 2 secondary antibody (Jackson Immuno Research, Ann Arbor, MI, USA) and counterstained with DAPI [74].

\subsection{Myeloid Derived Suppressor Cells (MDSC) and Regulatory T Cells ( $\left.T_{\text {reg }}\right)$}

Peripheral blood leukocytes (PBL) were purified from blood according to manufacturer instructions using Lympholyte-Mammal Cell Separation Media (Cedarlane, Burlington, ON, Canada). Livers and spleens were homogenized and filtered. Livers were then suspended in a 35\% Percoll gradient (Sigma Chemical, St Louis, MO, USA) and centrifugation for $15 \mathrm{~min}$ at $450 \mathrm{~g}$ at room temperature [75]. RBCs were lysed from spleens and livers prior to staining. Cells were stained using anti-CD4 (560782, clone: Rm4-5), anti-CD25 (551071, clone: PC61), anti-CD11b (557657, clone: 557657), anti-Ly6g (557657, clone: M1/70) (BD Biosciences), and anti-FoxP3 (17-5773-82, clone: FJK-16s, eBiosciences, San Diego, CA, USA) antibodies were diluted with 10\% goat serum in PBS and incubated for $45 \mathrm{~min}$ on ice. Cells were permeabilized using FoxP3 staining buffer set (eBioscience) following manufacturer instruction, except cells were incubated in the fix/permeabilization media overnight at $4{ }^{\circ} \mathrm{C}$. Anti-FoxP3 antibody was diluted in permeabilization buffer and added to cells for $30 \mathrm{~min}$ at room temperature. Staining was determined via flow cytometry. Statistical significance was determined via an unpaired $t$-test with $p<0.05$ (GraphPad Prism).

\subsection{Delayed Type Hypersensitivity (DTH)}

We compared DTH responses between EtOH and pair-fed mice after immunization with either OVA pDNA or influenza vaccine. OVA mice were immunized on Day 1 and boosted on Days 7 and 14 [76]. On Day 19, an eliciting dose was applied by GG to one ear. Ear thickness was measured $24 \mathrm{~h}$. In FLU 
DTH experiments, mice were immunized either IM or ID with $3 \mu \mathrm{g}$ influenza vaccine on Day 1 and were boosted on Day 21. One week later, vaccine was injected into one footpad and PBS into the other. Footpad thickness was measured at $24 \mathrm{~h}$. All data are presented as \% increase in thickness, determined using the equation:

$$
\left(\frac{\text { Treated Thickness }- \text { Control Thickness }}{\text { Control Thickness }}\right) \times 100
$$

Statistical significance was determined with an ANOVA followed by Fisher Exact Test with significance set at $p<0.05$ (GraphPad Prism).

\subsection{In Vivo Cytotoxic T Lymphocyte Killing Assay (CTL)}

To evaluate in vivo antigen specific lytic activity as a measure of CTL activity, mice were immunized with OVA pDNA via GG, IV, IM, and Hydro. Boosts were given on Days 7 (all), 14 (IV and IM), and 28 (IM) [77]. An in vivo CTL assay was performed five days after the last boost [76]. Percent lysis was calculated using the formula [76]:

$$
\% \text { lysis }=100 \times 1-\left(\frac{\frac{\text { CFSElow }}{\text { CFSEhigh of splenocytes from untreated mice }}}{\frac{\text { CFSElow }}{\text { CFSEhigh of splenocytes from immunized mice }}}\right)
$$

Statistical significance was determined via a one sample $t$-test and are compared to a theoretical mean of $100(p<0.05)$ (GraphPad Prism).

\subsection{Antibody Measurements}

ELISA plates were coated with influenza vaccine $(2 \mu \mathrm{g} / \mathrm{mL})$. Plates were blocked with $1 \%$ BSA in PBS for $1 \mathrm{~h}$, followed by biotinylated goat anti-mouse Fc receptor specific total IgG (Jackson Immuno, West Grove, PA, USA) for $2 \mathrm{~h}$ and Avidin-HRP (BD Pharmingen, San Jose, CA, USA) and then developed with TMB. The reaction was stopped immediately by adding $0.3 \mathrm{M}$ Sulfuric Acid. Data were converted via a standard curve generated with $\operatorname{IgG}$ (Sigma, I5381). Plates were read at $450 \mathrm{~nm}$. Statistical significance was determined via an unpaired $t$-test with significance set at $p<0.05$ (GraphPad Prism).

\section{Conclusions}

The long-term goals of these efforts are to better understand the effects of alcohol consumption on skin immunity, and to improve vaccine efficacy in alcohol consuming patients. We demonstrate that skin immunization obviates alcohol-induced immune inhibition in both liver sparing and liver-impairing mouse models of chronic alcohol consumption. Improvements in vaccine responses in heavy alcohol users could substantially increase vaccine efficacy in this large, well-defined population, with significant downstream public health benefits in terms of morbidity, mortality, and healthcare costs. This work supports the concept of ID vaccination as a standard of care for alcoholics. 


\section{Acknowledgments}

The authors would like to thank Patricia Eagan for her insightful discussions about physiological effects of alcohol abuse. This work was supported by NIH KO1AA017907 and K01AA017907-S1.

\section{Author Contributions}

Rhonda Brand-Designed, performed, analyzed and interpreted experiments, wrote the manuscript. John Mark Stottlemyer and Cara Donahue-Developed and performed laboratory assays. Rachel Cline-Performed laboratory assays. Jaideep Behari-Analyzed and interpreted all liver-related data. Louis Falo, Jr.--Designed experiments, interpreted results, wrote the manuscript.

\section{Conflicts of Interest}

The authors declare no conflict of interest.

\section{References}

1. Meyerholz, D.K.; Edsen-Moore, M.; McGill, J.; Coleman, R.A.; Cook, R.T.; Legge, K.L. Chronic alcohol consumption increases the severity of murine influenza virus infections. J. Immunol. 2008, 181, 641-648.

2. Lin, C.W.; Lin, C.C.; Mo, L.R.; Chang, C.Y.; Perng, D.S.; Hsu, C.C.; Lo, G.H.; Chen, Y.S.; Yen, Y.C.; Hu, J.T.; et al. Heavy alcohol consumption increases the incidence of hepatocellular carcinoma in hepatitis B virus-related cirrhosis. J. Hepatol. 2013, 58, 730-735.

3. Molina, P.E.; Bagby, G.J.; Nelson, S. Biomedical consequences of alcohol use disorders in the hiv-infected host. Curr. HIV Res. 2014, 12, 265-275.

4. Greenbaum, A.; Chaves, S.S.; Perez, A.; Aragon, D.; Bandyopadhyay, A.; Bennett, N.; Fowler, B.; Hancock, E.; Lynfield, R.; McDonald-Hamm, C.; et al. Heavy alcohol use as a risk factor for severe outcomes among adults hospitalized with laboratory-confirmed influenza, 2005-2012. Infection 2014, 42, 165-170.

5. Parlet, C.P.; Kavanaugh, J.S.; Horswill, A.R.; Schlueter, A.J. Chronic ethanol feeding increases the severity of Staphylococcus aureus skin infections by altering local host defenses. J Leukoc. Biol. 2015, 97, 769-778.

6. Mason, C.M.; Dobard, E.; Zhang, P.; Nelson, S. Alcohol exacerbates murine pulmonary tuberculosis. Infecti. Immun. 2004, 72, 2556-2563.

7. Shellito, J.E.; Quan Zheng, M.; Ye, P.; Ruan, S.; Shean, M.K.; Kolls, J. Effect of alcohol consumption on host release of interleukin-17 during pulmonary infection with Klebsiella pneumoniae. Alcohol. Clin. Exp. Res. 2001, 25, 872-881.

8. Grau, I.; Ardanuy, C.; Calatayud, L.; Schulze, M.H.; Liñares, J.; Pallares, R. Smoking and alcohol abuse are the most preventable risk factors for invasive pneumonia and other pneumococcal infections. Int. J. Infect. Dis. 2014, 25, 59-64.

9. Mendenhall, C.; Roselle, G.A.; Lybecker, L.A.; Marshall, L.E.; Grossman, C.J.; Myre, S.A.; Weesner, R.E.; Morgan, D.D. Hepatitis B vaccination. Response of alcoholic with and without liver injury. Dig. Dis. Sci. 1988, 33, 263-269. 
10. Nalpas, B.; Thepot, V.; Driss, F.; Pol, S.; Courouce, A.M.; Saliou, P.; Berthelot, P. Secondary immune response to hepatitis B virus vaccine in alcoholics. Alcohol. Clin. Exp. Res. 1993, 17, 295-298.

11. Benin, A.L.; O’Brien, K.L.; Watt, J.P.; Reid, R.; Zell, E.R.; Katz, S.; Donaldson, C.; Parkinson, A.; Schuchat, A.; Santosham, M.; et al. Effectiveness of the 23-valent polysaccharide vaccine against invasive pneumococcal disease in navajo adults. J. Infect. Dis. 2003, 188, 81-89.

12. Porretta, E.; Happel, K.I.; Teng, X.S.; Ramsay, A.; Mason, C.M. The impact of alcohol on BCG-induced immunity against Mycobacterium tuberculosis. Alcohol. Clin. Exp. Res. 2012, 36, 310-317.

13. Rosman, A.S.; Basu, P.; Galvin, K.; Lieber, C.S. Efficacy of a high and accelerated dose of hepatitis B vaccine in alcoholic patients: A randomized clinical trial. Am. J. Med. 1997, 103, 217-222.

14. De Maria, N.; Idilman, R.; Colantoni, A.; van Thiel, D.H. Increased effective immunogenicity to high-dose and short-interval hepatitis B virus vaccination in individuals with chronic hepatitis without cirrhosis. J. Viral Hepat. 2001, 8, 372-376.

15. Nagafuchi, S.; Kashiwagi, S.; Okada, K.; Anzai, K.; Nakamura, M.; Nishimura, Y.; Sasazuki, T.; Niho, Y. Reversal of nonresponders and postexposure prophylaxis by intradermal hepatitis B vaccination in japanese medical personnel. JAMA 1991, 265, 2679-2683.

16. Rahman, F.; Dahmen, A.; Herzog-Hauff, S.; Bocher, W.O.; Galle, P.R.; Lohr, H.F. Cellular and humoral immune responses induced by intradermal or intramuscular vaccination with the major hepatitis B surface antigen. Hepatology 2000, 31, 521-527.

17. Combadiere, B.; Liard, C. Transcutaneous and intradermal vaccination. Hum. Vaccine 2011, 7, 811-827.

18. Messaoudi, I.; Pasala, S.; Grant, K. Could moderate alcohol intake be recommended to improve vaccine responses? Expert Rev. Vaccines 2014, 13, 817-819.

19. Dehne, N.E.; Mendenhall, C.L.; Roselle, G.A.; Grossman, C.J. Cell-mediated immune responses associated with short term alcohol intake: Time course and dose dependency. Alcohol. Clin. Exp. Res. 1989, 13, 201-205.

20. Mendenhall, C.L.; Theus, S.A.; Roselle, G.A.; Grossman, C.J.; Rouster, S.D. Biphasic in vivo immune function after low-versus high-dose alcohol consumption. Alcohol 1997, 14, 255-260.

21. Lau, A.H.; Szabo, G.; Thomson, A.W. Antigen-presenting cells under the influence of alcohol. Trends Immunol. 2009, 30, 13-22.

22. Brand, R.M.; Jendrzejewski, J.L. Chronic ethanol ingestion alters xenobiotic absorption through the skin: Potential role of oxidative stress. Food Chem. Toxicol. 2008, 46, 1940-1948.

23. Edsen-Moore, M.R.; Fan, J.; Ness, K.J.; Marietta, J.R.; Cook, R.T.; Schlueter, A.J. Effects of chronic ethanol feeding on murine dendritic cell numbers, turnover rate, and dendropoiesis. Alcohol. Clin. Exp. Res. 2008, 32, 1309-1320.

24. Ness, K.J.; Fan, J.; Wilke, W.W.; Coleman, R.A.; Cook, R.T.; Schlueter, A.J. Chronic ethanol consumption decreases murine langerhans cell numbers and delays migration of langerhans cells as well as dermal dendritic cells. Alcohol. Clin. Exp. Res. 2008, 32, 657-668.

25. D’Souza El-Guindy, N.B.; Kovacs, E.J.; de Witte, P.; Spies, C.; Littleton, J.M.; de Villiers, W.J.; Lott, A.J.; Plackett, T.P.; Lanzke, N.; Meadows, G.G. Laboratory models available to study alcohol-induced organ damage and immune variations: Choosing the appropriate model. Alcohol. Clin. Exp. Res. 2010, 34, 1489-1511. 
26. Abdallah, R.M.; Starkey, J.R.; Meadows, G.G. Toxicity of chronic high alcohol intake on mouse natural killer cell activity. Res. Commun. Chem. Pathol. Pharmacol. 1988, 59, 245-258.

27. Cook, R.T.; Schlueter, A.J.; Coleman, R.A.; Tygrett, L.; Ballas, Z.K.; Jerrells, T.R.; Nashelsky, M.B.; Ray, N.B.; Haugen, T.H.; Waldschmidt, T.J. Thymocytes, pre-B cells, and organ changes in a mouse model of chronic ethanol ingestion-Absence of subset-specific glucocorticoid-induced immune cell loss. Alcohol. Clin. Exp. Res. 2007, 31, 1746-1758.

28. Lieber, C.S.; DeCarli, L.M. The feeding of alcohol in liquid diets: Two decades of applications and 1982 update. Alcohol. Clin. Exp. Res. 1982, 6, 523-531.

29. Das, S.K.; Vasudevan, D.M. Alcohol-induced oxidative stress. Life Sci. 2007, 81, 177-187.

30. Cresci, G.A.; Bush, K.; Nagy, L.E. Tributyrin supplementation protects mice from acute ethanol-induced gut injury. Alcohol. Clin. Exp. Res. 2014, 38, 1489-1501.

31. Bode, C.; Bode, J.C. Activation of the innate immune system and alcoholic liver disease: Effects of ethanol per se or enhanced intestinal translocation of bacterial toxins induced by ethanol? Alcohol. Clin. Exp. Res. 2005, 29, 166S-171S.

32. Purohit, V.; Bode, J.C.; Bode, C.; Brenner, D.A.; Choudhry, M.A.; Hamilton, F.; Kang, Y.J.; Keshavarzian, A.; Rao, R.; Sartor, R.B.; et al. Alcohol, intestinal bacterial growth, intestinal permeability to endotoxin, and medical consequences: Summary of a symposium. Alcohol 2008, 42, 349-361.

33. Park, P.H.; Thakur, V.; Pritchard, M.T.; McMullen, M.R.; Nagy, L.E. Regulation of kupffer cell activity during chronic ethanol exposure: Role of adiponectin. J. Gastroenterol. Hepatol. 2006, 21, S30-S33.

34. Lieber, C.S. Alcoholic fatty liver: Its pathogenesis and mechanism of progression to inflammation and fibrosis. Alcohol 2004, 34, 9-19.

35. Brand, R.M.; Jendrzejewski, J.L.; Charron, A.R. Potential mechanisms by which a single drink of alcohol can increase transdermal absorption of topically applied chemicals. Toxicology 2007, 235, 141-149.

36. Van Epps, E.; Husby, G.; Williams, R.C., Jr.; Strickland, R.G. Liver disease-A prominent cause of serum ige elevation. Clin. Exp. Immunol. 1976, 23, 444-450.

37. Wang, H.J.; Gao, B.; Zakhari, S.; Nagy, L.E. Inflammation in alcoholic liver disease. Annu. Rev. Nutr. 2012, 32, 343-368.

38. Esterbauer, H.; Schaur, R.J.; Zollner, H. Chemistry and biochemistry of 4-hydroxynonenal, malonaldehyde and related aldehydes. Free Radic. Biol. Med. 1991, 11, 81-128.

39. Wang, H.; Chan, Y.-L.; Li, T.-L.; Bauer, B.A.; Hsia, S.; Wang, C.-H.; Huang, J.-S.; Wang, H.-M.; Yeh, K.-Y.; Huang, T.-H.; et al. Reduction of splenic immunosuppressive cells and enhancement of anti-tumor immunity by synergy of fish oil and selenium yeast. PLoS ONE 2013, 8, e52912.

40. Gabrilovich, D.I.; Nagaraj, S. Myeloid-derived suppressor cells as regulators of the immune system. Nat. Rev. Immunol. 2009, 9, 162-174.

41. Zhu, B.; Bando, Y.; Xiao, S.; Yang, K.; Anderson, A.C.; Kuchroo, V.K.; Khoury, S.J. $\mathrm{Cd} 11 \mathrm{~b}^{+} \mathrm{ly}^{-} \mathrm{c}(\mathrm{hi})$ suppressive monocytes in experimental autoimmune encephalomyelitis. J. Immunol. 2007, 179, 5228-5237. 
42. Chen, S.; Akbar, S.M.; Abe, M.; Hiasa, Y.; Onji, M. Immunosuppressive functions of hepatic myeloid-derived suppressor cells of normal mice and in a murine model of chronic hepatitis B virus. Clin. Exp. Immunol. 2011, 166, 134-142.

43. Wu, X.; He, Y.; Falo, L.D., Jr.; Hui, K.M.; Huang, L. Regression of human mammary adenocarcinoma by systemic administration of a recombinant gene encoding the hflex-trail fusion protein. Mol. Ther. 2001, 3, 368-374.

44. Brand, R.M. University of Pittsburgh,. Pittsburgh, PA, USA. Unpublished work, 2009.

45. Lau, A.H.; Abe, M.; Thomson, A.W. Ethanol affects the generation, cosignaling molecule expression, and function of plasmacytoid and myeloid dendritic cell subsets in vitro and in vivo. J. Leukoc. Biol. 2006, 79, 941-953.

46. Fan, J.; Edsen-Moore, M.R.; Turner, L.E.; Cook, R.T.; Legge, K.L.; Waldschmidt, T.J.; Schlueter, A.J. Mechanisms by which chronic ethanol feeding limits the ability of dendritic cells to stimulate T-cell proliferation. Alcohol. Clin. Exp. Res. 2011, 35, 47-59.

47. Parlet, C.P.; Schlueter, A.J. Mechanisms by which chronic ethanol feeding impairs the migratory capacity of cutaneous dendritic cells. Alcohol. Clin. Exp. Res. 2013, 37, 2098-2107.

48. Heinz, R.; Waltenbaugh, C. Ethanol consumption modifies dendritic cell antigen presentation in mice. Alcohol. Clin. Exp. Res. 2007, 31, 1759-1771.

49. Kim, J.H. Effect of biphenyl dimethyl dicarboxylate on the cellular and nonspecific immunotoxicity by ethanol in mice. Biol. Pharm. Bull. 2000, 23, 1206-1211.

50. Thyssen, J.P.; Nielsen, N.H.; Linneberg, A. The association between alcohol consumption and contact sensitization in danish adults: The glostrup allergy study. Br. J. Dermatol. 2008, 158, 306-312.

51. Geissler, M.; Gesien, A.; Wands, J.R. Inhibitory effects of chronic ethanol consumption on cellular immune responses to hepatitis $\mathrm{C}$ virus core protein are reversed by genetic immunizations augmented with cytokine-expressing plasmids. J. Immunol. 1997, 159, 5107-5113.

52. Encke, J.; Wands, J.R. Ethanol inhibition: The humoral and cellular immune response to hepatitis C virus ns5 protein after genetic immunization. Alcohol. Clin. Exp. Res. 2000, 24, 1063-1069.

53. Aloman, C.; Gehring, S.; Wintermeyer, P.; Kuzushita, N.; Wands, J.R. Chronic ethanol consumption impairs cellular immune responses against HCV ns5 protein due to dendritic cell dysfunction. Gastroenterology 2007, 132, 698-708.

54. Ito, T.; Amakawa, R.; Kaisho, T.; Hemmi, H.; Tajima, K.; Uehira, K.; Ozaki, Y.; Tomizawa, H.; Akira, S.; Fukuhara, S. Interferon-alpha and interleukin-12 are induced differentially by toll-like receptor 7 ligands in human blood dendritic cell subsets. J. Exp. Med. 2002, 195, 1507-1512.

55. Cheong, H.J.; Song, J.Y.; Park, J.W.; Yeon, J.E.; Byun, K.S.; Lee, C.H.; Cho, H.I.; Kim, T.G.; Kim, W.J. Humoral and cellular immune responses to influenza vaccine in patients with advanced cirrhosis. Vaccine 2006, 24, 2417-2422.

56. Hagedorn, H.J.; Rettmann, N.A.; Dieperink, E.W.; Durfee, J.; Aqel, B. Antibody response to hepatitis B vaccine in substance use disorder patients. Drug Alcohol. Depend. 2010, 107, 39-43.

57. Arnou, R.; Icardi, G.; de Decker, M.; Ambrozaitis, A.; Kazek, M.P.; Weber, F.; van Damme, P. Intradermal influenza vaccine for older adults: A randomized controlled multicenter phase III study. Vaccine 2009, 27, 7304-7312. 
58. Fabrizi, F.; Dixit, V.; Messa, P.; Martin, P. Intradermal vs. intramuscular vaccine against hepatitis B infection in dialysis patients: A meta-analysis of randomized trials. J. Viral Hepat. 2011, 18, 730-737.

59. Dhillon, S.; Moore, C.; Li, S.D.; Aziz, A.; Kakar, A.; Dosanjh, A.; Beesla, A.; Murphy, L.; van Thiel, D.H. Efficacy of high-dose intra-dermal hepatitis B virus vaccine in previous vaccination non-responders with chronic liver disease. Dig. Dis. Sci. 2012, 57, 215-220.

60. Morelon, E.; Pouteil Noble, C.; Daoud, S.; Cahen, R.; Goujon-Henry, C.; Weber, F.; Laurent, P.E.; Kaiserlian, D.; Nicolas, J.F. Immunogenicity and safety of intradermal influenza vaccination in renal transplant patients who were non-responders to conventional influenza vaccination. Vaccine 2010, 28, 6885-6890.

61. Latif, O.; Peterson, J.D.; Waltenbaugh, C. Alcohol-mediated polarization of type 1 and type 2 immune responses. Front. Biosci. 2002, 7, a135-a147.

62. Eken, A.; Ortiz, V.; Wands, J.R. Ethanol inhibits antigen presentation by dendritic cells. Clin. Vaccine Immunol. 2011, 18, 1157-1166.

63. Waldschmidt, T.J.; Cook, R.T.; Kovacs, E.J. Alcohol and inflammation and immune responses: Summary of the 2005 alcohol and immunology research interest group (AIRIG) meeting. Alcohol 2006, 38, 121-125.

64. Trinchieri, G. Interleukin-12 and its role in the generation of th1 cells. Immunol. Today 1993, 14, $335-338$.

65. Iclozan, C.; Antonia, S.; Chiappori, A.; Chen, D.-T.; Gabrilovich, D. Therapeutic regulation of myeloid-derived suppressor cells and immune response to cancer vaccine in patients with extensive stage small cell lung cancer. Cancer Immunol. Immunother. 2013, 62, 909-918.

66. Sui, Y.; Hogg, A.; Wang, Y.; Frey, B.; Yu, H.; Xia, Z.; Venzon, D.; McKinnon, K.; Smedley, J.; Gathuka, M.; et al. Vaccine-induced myeloid cell population dampens protective immunity to SIV. J. Clin. Investig. 2014, 124, 2538-2549.

67. Zhang, H.; Meadows, G. Chronic alcohol consumption enhances myeloid-derived suppressor cells in b16bl6 melanoma-bearing mice. Cancer Immunol. Immunother. 2010, 59, 1151-1159.

68. Chaudhry, K.K.; Samak, G.; Shukla, P.K.; Mir, H.; Gangwar, R.; Manda, B.; Isse, T.; Kawamoto, T.; Salaspuro, M.; Kaihovaara, P.; et al. Aldh2 deficiency promotes ethanol-induced gut barrier dysfunction and fatty liver in mice. Alcohol. Clin. Exp. Res. 2015, 39, 1465-1475.

69. Cohen, J.I.; Roychowdhury, S.; McMullen, M.R.; Stavitsky, A.B.; Nagy, L.E. Complement and alcoholic liver disease: Role of $\mathrm{clq}$ in the pathogenesis of ethanol-induced liver injury in mice. Gastroenterology 2010, 139, 664-674.

70. Larosche, I.; Choumar, A.; Fromenty, B.; Letteron, P.; Abbey-Toby, A.; van Remmen, H.; Epstein, C.J.; Richardson, A.; Feldmann, G.; Pessayre, D.; et al. Prolonged ethanol administration depletes mitochondrial DNA in mnsod-overexpressing transgenic mice, but not in their wild type littermates. Toxicol. Appl. Pharmacol. 2009, 234, 326-338.

71. Larregina, A.T.; Morelli, A.E.; Tkacheva, O.; Erdos, G.; Donahue, C.; Watkins, S.C.; Thomson, A.W.; Falo, L.D., Jr. Highly efficient expression of transgenic proteins by naked DNA-transfected dendritic cells through terminal differentiation. Blood 2004, 103, 811-819.

72. Kleiner, D.E.; Brunt, E.M.; van Natta, M.; Behling, C.; Contos, M.J.; Cummings, O.W.; Ferrell, L.D.; Liu, Y.C.; Torbenson, M.S.; Unalp-Arida, A.; et al. Design and validation of a histological scoring system for nonalcoholic fatty liver disease. Hepatology 2005, 41, 1313-1321. 
73. Liu, D.; Li, C.; Chen, Y.; Burnett, C.; Liu, X.Y.; Downs, S.; Collins, R.D.; Hawiger, J. Nuclear import of proinflammatory transcription factors is required for massive liver apoptosis induced by bacterial lipopolysaccharide. J. Biol. Chem. 2004, 279, 48434-48442.

74. Roychowdhury, S.; McMullen, M.R.; Pritchard, M.T.; Hise, A.G.; van Rooijen, N.; Medof, M.E.; Stavitsky, A.B.; Nagy, L.E. An early complement-dependent and TLR-4-independent phase in the pathogenesis of ethanol-induced liver injury in mice. Hepatology 2009, 49, 1326-1334.

75. Arai, K.; Liu, Z.X.; Lane, T.; Dennert, G. Ip-10 and mig facilitate accumulation of T cells in the virus-infected liver. Cell Immunol. 2002, 219, 48-56.

76. Mathers, A.R.; Tckacheva, O.A.; Janelsins, B.M.; Shufesky, W.J.; Morelli, A.E.; Larregina, A.T. In vivo signaling through the neurokinin 1 receptor favors transgene expression by langerhans cells and promotes the generation of th1- and tc1-biased immune responses. J. Immunol. 2007, 178, 7006-7017.

77. Morel, P.A.; Falkner, D.; Plowey, J.; Larregina, A.T.; Falo, L.D. DNA immunisation: Altering the cellular localisation of expressed protein and the immunisation route allows manipulation of the immune response. Vaccine 2004, 22, 447-456.

(C) 2015 by the authors; licensee MDPI, Basel, Switzerland. This article is an open access article distributed under the terms and conditions of the Creative Commons Attribution license (http://creativecommons.org/licenses/by/4.0/). 